\title{
Treatment of venous thromboembolism in a 22-year-old woman taking an oral contraceptive pill
}

\author{
Daniel M. Shafran MD, Cynthia Wu MD
}

Cite as: CMAJ 2017 November 27;189:E1459-60. doi: 10.1503/cmaj.170398

A previously healthy 22-year-old woman presented to the emergency department with shortness of breath. She had been taking a combined oral contraceptive pill since the age of 17 years. Computed tomographic pulmonary angiography confirmed the diagnosis of pulmonary embolism and she was started on rivaroxaban. She is seeing her family physician for follow-up.

\section{Has the patient been prescribed an appropriate anticoagulant?}

Direct oral anticoagulants are the preferred initial treatment for acute venous thromboembolism in ambulatory patients without cancer. Several randomized controlled trials (RCTs) on acute venous thromboembolism have found direct oral anticoagulants to be noninferior to standard therapy - consisting of enoxaparin (lowmolecular-weight heparin) followed by warfarin (vitamin $\mathrm{K}$ antagonist) - in reducing recurrent venous thromboembolism. ${ }^{1,2}$ Safety outcomes of dabigatran and rivaroxaban showed no significant difference in bleeding risk compared with standard therapy, while apixaban and edoxaban reduced the absolute risk of major or clinically relevant nonmajor bleeding by $5.4 \%$ and $1.8 \%$, respectively. ${ }^{1-4}$

Direct oral anticoagulants are more convenient than vitamin $\mathrm{K}$ antagonists, because they do not require laboratory monitoring and there are fewer drug interactions and no known food interactions. However, they are more expensive than vitamin $\mathrm{K}$ antagonists and dependent on renal clearance. The American College of Chest Physicians guideline recommends direct oral anticoagulants over vitamin $\mathrm{K}$ antagonists as first-line therapy for venous thromboembolism in patients who do not have cancer. ${ }^{5}$

Should the patient stop taking the oral contraceptive pill? It appears safe to continue the combined oral contraceptive pill in patients with acute venous thromboembolism who are receiving anticoagulation. A subgroup analysis of 1888 women younger than 60 years of age who had been enrolled in an RCT of rivaroxaban versus standard therapy for acute venous thromboembolism found hormonal therapy was not associated with increased risk of recurrent venous thromboembolism during therapeutic anticoagulation. ${ }^{6}$
In addition, continuing the oral contraceptive pill is a simple and effective method to ensure that women who receive anticoagulation also receive contraception, which decreases the risk of fetal exposure to oral anticoagulation. Both vitamin $\mathrm{K}$ antagonists and direct oral anticoagulants are contraindicated in pregnancy. ${ }^{5}$

Immediately stopping the oral contraceptive pill in a woman who requires anticoagulation with direct oral anticoagulants may substantially worsen menorrhagia, potentially leading to decreased quality of life and iron deficiency anemia. Direct oral anticoagulants of the factor $\mathrm{Xa}$ inhibitor group appear to increase menorrhagia more than vitamin $\mathrm{K}$ antagonists. In the aforementioned analysis, abnormal uterine bleeding doubled in women who were receiving rivaroxaban compared with standard therapy, with a hazard ratio of $2.13(95 \%$ confidence interval [Cl] 1.57-2.89). ${ }^{6} \mathrm{~A}$ subgroup analysis of a large apixaban trial found a similar effect. ${ }^{7}$ The mechanism is unknown.

If anticoagulation is stopped, estrogen should be avoided thereafter to decrease the risk of recurrent venous thromboembolism. Progestin-only contraceptives can be considered.

\section{For how long should anticoagulation be continued?}

Anticoagulation for acute venous thromboembolism should be continued for a minimum of three months; the decision to extend anticoagulation depends on the risk of recurrent venous thromboembolism, bleeding and patient preference.

Risk of recurrence in patients with venous thromboembolism provoked by a nonsurgical risk factor, such as estrogen, is estimated at $5 \%$ within one year and $15 \%$ within five years when anticoagulation is stopped after a minimum of three months. ${ }^{5}$ Although the annual risk of bleeding in patients on anticoagulation without risk factors is estimated at $0.8 \%$, the case fatality from major bleeding (11.3\%) is greater than from recurrent venous thromboembolism (3.6\%). 5,8 Therefore, the American College of Chest Physicians guideline recommends stopping anticoagulation after three months for venous thromboembolism that is provoked by a reversible risk factor. ${ }^{5}$

Absent other risk factors, venous thromboembolism in this setting may be considered to have been provoked by the oral contraceptive pill. Anticoagulation may be stopped after three 
months in patients who are no longer taking the oral contraceptive pill, but should be continued indefinitely as long as oral contraception is still being used.

\section{Does the patient require anticoagulation if planning pregnancy?}

It is recommended that pregnant women with a history of estrogen-provoked venous thromboembolism receive antepartum prophylaxis, and all pregnant women with previous venous thromboembolism should receive postpartum prophylaxis for six weeks. ${ }^{5}$

A retrospective cohort study of more than 8000 women found that women whose first venous thromboembolism was associated with pregnancy or the postpartum period had a higher risk of recurrence during a subsequent pregnancy than women with unprovoked venous thromboembolism $(4.5 \% \mathrm{v}$. 2.7\%; relative risk 1.71; 95\% $\mathrm{Cl} 1.0-2.8) .{ }^{9}$ The American College of Chest Physicians considers that women with a history of venous thromboembolism related to estrogen have a moderate to high risk of recurrence. ${ }^{5}$

Little evidence guides peripartum prophylaxis. A systematic review of low-molecular-weight heparin evaluated 64 studies reporting 2777 pregnancies; of 1436 women receiving thromboprophylaxis with low-molecular-weight heparin, only $13(0.9 \%)$ developed venous thromboembolism. There were no maternal deaths and a $1.98 \%$ rate of substantial bleeding, mostly owing to obstetric causes. ${ }^{10}$ Because of these findings and low-molecularweight heparin's long record of safety, the American College of Chest Physicians guideline recommends low-molecular-weight heparin for thromboprophylaxis in pregnancy. ${ }^{5}$

In the first six weeks postpartum, low-molecular-weight heparin or warfarin may be used as neither is excreted into breast milk; conversely, the excretion of direct oral anticoagulants in breast milk is unknown, so they remain contraindicated. ${ }^{11}$ Referral to a hematologist or specialist in obstetric medicine and a specialist in high-risk obstetrics is suggested for women in whom venous thromboembolism prophylaxis may be warranted during or immediately after pregnancy.

\section{Case revisited}

Further inquiry showed the patient was using the oral contraceptive pill solely for contraception and did not want indefinite anticoagulation. The combined oral contraceptive pill was continued for several weeks during anticoagulation, at which time the patient received an intrauterine device and the oral contraceptive pill was stopped. Because estrogen exposure had been discontinued, rivaroxaban was continued for three months, then stopped. The patient was informed that she will require referral to an expert in obstetric medicine for prenatal planning, as well as thromboprophylaxis with low-molecular-weight heparin, during pregnancy and for six weeks postpartum.

\section{References}

1. Agnelli G, Buller HR, Cohen A, et al.; AMPLIFY Investigators. Oral apixaban for the treatment of acute venous thromboembolism. N Engl J Med 2013;369:799-808.

2. EINSTEIN-PE Investigators; Büller HR, Prins MH, Lensin AW, et al. Oral rivaroxaban for the treatment of symptomatic pulmonary embolism. N Engl J Med 2012; 366:1287-97.

3. Schulman S, Kearon C, Kakkar AK, et al.; RE-COVER Study Group. Dabigatran versus warfarin in the treatment of acute venous thromboembolism. N Engl J Med 2009;361:2342-52.

4. Hokusai-VTE Investigators; Büller HR, Décousus H, Grosso MA, et al. Edoxaban versus warfarin for the treatment of acute venous thromboembolism. $N$ Engl J Med 2013;369:1406-15.

5. Kearon C, Akl EA, Ornelas J, et al. Antithrombotic therapy for VTE disease: Chest guideline and expert panel report. Chest 2016;149:315-52.

6. Martinelli I, Lensing AWA, Middeldorp S, et al. Recurrent venous thromboembolism and abnormal uterine bleeding with anticoagulant and hormone therapy use. Blood 2016;127:1417-25.

7. Brekelmans MPA, Scheres LJJ, Bleker SM, et al. Abnormal vaginal bleeding in women with venous thromboembolism treated with apixaban or warfarin. Thromb Haemost 2017;117:809-15.

8. Carrier M, Le Gal G, Wells PS, et al. Systematic review: case-fatality rates of recurrent venous thromboembolism and major bleeding events among patients treated for venous thromboembolism. Ann Intern Med 2010;152:578-89.

9. White $\mathrm{RH}$, Chans WS, Zhou H, et al. Recurrent venous thromboembolism after pregnancy-associated versus unprovoked thromboembolism. Thromb Haemost 2008;100:246-52.

10. Greer IA, Nelson-Piercy C. Low-molecular-weight heparins for thromboprophylaxis and treatment of venous thromboembolism in pregnancy: a systematic review of safety and efficacy. Blood 2005;106:401-7.

11. Burnett AE, Mahan CE, Vazquez SR, et al. Guidance for the practical management of the direct oral anticoagulants (DOACS) in VTE treatment. J Thromb Thrombolysis 2016;41:206-32.

Competing interests: Cynthia Wu reports speaker's fees and advisory board honoraria from LEO Pharma and Pfizer, outside the submitted work. No other competing interests were declared.

This article has been peer reviewed.

The clinical scenario is fictional.

Affiliations: Department of Medicine (Shafran, Wu); Division of Hematology $(\mathrm{Wu})$, University of Alberta, Edmonton, Alta.

Contributors: Both authors contributed to the conception and design of the work, drafted the manuscript, revised it critically for important intellectual content, gave final approval of the version to be published and agreed to be accountable for all aspects of the work.

Correspondence to: Cynthia Wu, cwu@ualberta.ca

Decisions is a series that focuses on practical evidence-based approaches to common presentations in primary care. The articles address key decisions that a clinician may encounter during initial assessment. The information presented can usually be covered in a typical primary care appointment. Articles should be no longer than 650 words, may include one box, figure or table and should begin with a very brief description ( 75 words or less) of the clinical situation. The decisions addressed should be presented in the form of questions. A box providing helpful resources for the patient or physician is encouraged. 